\title{
A New Modification of Adomian Decomposition Method for Volterra Integral Equations of the Second Kind
}

\author{
Lie-jun Xie \\ Department of Mathematics, Faculty of Science, Ningbo University, Ningbo, Zhejiang 315211, China \\ Correspondence should be addressed to Lie-jun Xie; xieliejun@nbu.edu.cn
}

Received 5 September 2013; Accepted 4 November 2013

Academic Editor: Roberto Barrio

Copyright (C) 2013 Lie-jun Xie. This is an open access article distributed under the Creative Commons Attribution License, which permits unrestricted use, distribution, and reproduction in any medium, provided the original work is properly cited.

We propose a new modification of the Adomian decomposition method for Volterra integral equations of the second kind. By the Taylor expansion of the components apart from the zeroth term of the Adomian series solution, this new technology overcomes the problems arising from the previous decomposition method. The validity and applicability of the new technique are illustrated through several linear and nonlinear equations by comparing with the standard decomposition method and the modified decomposition method. The results obtained indicate that the new modification is effective and promising.

\section{Introduction}

The Adomian decomposition method (ADM) $[1,2]$ has been efficiently used to solve linear and nonlinear problems such as differential equations and integral equations. The method provides the solution as an infinite series in which each term can be easily determined. The rapid convergence of the series obtained by this method is thoroughly discussed by Cherruault et al. [3].

Recently, Wazwaz [4] proposed a reliable modified technique of $\mathrm{ADM}$ that accelerates the rapid convergence of decomposition series solution. The modified decomposition needs only a slight variation from the standard decomposition method. Although the modified decomposition method may provide the exact solution by using two iterations only and sometimes without using the so-called Adomian polynomials, its effectiveness is based on the assumption that the function $f$ can be divided into two parts, and thus the success of the modified method depends on the proper choice of $f_{1}$ and $f_{2}$.

More recently, another new modification of decomposition method was proposed by Wazwaz and El-Sayed [5]. In the new modification, the process of dividing $f$ into two components was replaced by a Taylor series of infinite components. The ideas have been shown to be computationally efficient in applying the proposed technique in several differential and integral equations. However, as will be seen from the examples below, the modified decomposition method will sometimes encounter obstacles to obtain the exact solution. After that, several authors further proposed expressing the function $f$ by the orthogonal polynomial series, such as Chebyshev series [6], Legendre series [7, 8], and Jacobi and Gegenbauer series [9].

In this paper, we introduce a new modification of decomposition method and make further progress beyond the achievements made so far in this regard. Several examples concerning integral equations are tested, and the results suggest that this new idea proposes a promising tool for the computation of integral equations both linear and nonlinear.

\section{Description of the New Modification}

Since the beginning of the 1980s, the Adomian decomposition method has been applied to a wide class of integral equations. To illustrate the procedure, consider the following Volterra integral equations of the second kind given by

$$
\begin{aligned}
u(x)= & f(x) \\
& +\lambda \int_{a}^{x} K(x, t)(L(u(t))+N(u(t))) \mathrm{d} t, \quad \lambda \neq 0,
\end{aligned}
$$


where the kernel $K(x, t)$ and the function $f(x)$ are given realvalued functions, $\lambda$ is a parameter, $L(u(x))$ and $N(u(x))$ are linear and nonlinear operators of $u(x)$, respectively, and the unknown function $u(x)$ is the solution to be determined.

In the decomposition method, we usually express the solution of (1) in a series form defined by

$$
u(x)=\sum_{k=0}^{+\infty} u_{k}(x) .
$$

Moreover, the decomposition method identifies the nonlinear term $N(u(x))$ by the decomposition series

$$
N(u(x))=\sum_{k=0}^{+\infty} A_{k}(x)
$$

where $A_{k}(x)$ is the so-called Adomian polynomials, which can be evaluated by the following formula $[10,11]$ :

$$
A_{n}=\frac{1}{n !} \frac{\mathrm{d}^{n}}{\mathrm{~d} \lambda^{n}} N\left(\sum_{i=0}^{n} \lambda^{i} u_{i}\right), \quad n=0,1,2, \ldots
$$

Substituting (2) and (3) into both sides of (1) gives

$$
\begin{aligned}
\sum_{k=0}^{+\infty} u_{k}(x)= & f(x) \\
& +\lambda \int_{a}^{x} K(x, t)\left[L\left(\sum_{k=0}^{+\infty} u_{k}(t)\right)+\sum_{k=0}^{+\infty} A_{k}(t)\right] \mathrm{d} t .
\end{aligned}
$$

In the following we outline the basic feature of the standard decomposition method $[1,2]$ and the modified decomposition method $[4,5]$.

The Standard Adomian Decomposition Method (SADM). By the standard decomposition method, the components $u_{0}(x)$, $u_{1}(x), u_{2}(x), \ldots$ of the solution $u(x)$ of (1), are completely determined in the following recurrence manner:

$$
\begin{gathered}
u_{0}(x)=f(x), \\
u_{k+1}(x)=\lambda \int_{a}^{x} K(x, t)\left(L\left(u_{k}\right)+A_{k}\right) \mathrm{d} t, \quad k \geq 0 .
\end{gathered}
$$

Having determined the components $u_{0}(x), u_{1}(x), u_{2}(x), \ldots$, the solution $u(x)$ in a series form defined by (2) follows immediately.

The Modified Decomposition Method 1 (MADM1). The standard decomposition method by Adomian $[1,2]$ was modified by Wazwaz [4]. The modification is based on the assumption that the function $f(x)$ can be divided into two parts, namely, $f_{1}(x)$ and $f_{2}(x)$. Under this assumption we set

$$
f(x)=f_{1}(x)+f_{2}(x) .
$$

Accordingly, a slight variation was proposed only for the components $u_{0}(x)$ and $u_{1}(x)$. The suggestion was that only the part $f_{1}(x)$ will be assigned to the zeroth component $u_{0}(x)$, whereas the remaining part $f_{2}(x)$ will be combined with the other terms given into $(6 \mathrm{~b})$ to define $u_{1}(x)$. Consequently, the modified recursive relation

$$
\begin{gathered}
u_{0}(x)=f_{1}(x), \\
u_{1}(x)=f_{2}(x)+\lambda \int_{a}^{x} K(x, t)\left(L\left(u_{0}\right)+A_{0}\right) \mathrm{d} t, \\
u_{k+1}(x)=\lambda \int_{a}^{x} K(x, t)\left(L\left(u_{k}\right)+A_{k}\right) \mathrm{d} t, \quad k \geq 1,
\end{gathered}
$$

was developed.

It is important to note here that there were some conclusions made in [4]. First, the slight variation in reducing the number of terms of $u_{0}$ will result in a reduction of the computational work and will accelerate the convergence. Second, this slight variation in the definition of the components $u_{0}$ and $u_{1}$ may provide the solution by using two iterations only. Third, there is no need sometimes to evaluate the so-called Adomian polynomials required for the nonlinear equations.

The Modified Decomposition Method 2 (MADM2). In [5], it is pointed that sometimes it may be useful to express $f(x)$ in Taylor series as follows:

$$
f(x)=\sum_{i=0}^{+\infty} f_{i}(x)
$$

Meanwhile, a new recursive relationship in the following form can be obtained:

$$
\begin{gathered}
u_{0}(x)=f_{0}(x), \\
u_{1}(x)=f_{1}(x)+\lambda \int_{a}^{x} K(x, t)\left(L\left(u_{0}\right)+A_{0}\right) \mathrm{d} t, \\
u_{k+1}(x)=f_{k+1}(x) \\
+\lambda \int_{a}^{x} K(x, t)\left(L\left(u_{k}\right)+A_{k}\right) \mathrm{d} t, \quad k \geq 1 .
\end{gathered}
$$

According to (10), the terms $u_{0}(x), u_{1}(x), u_{2}(x), \ldots$ of the solution $u(x)$ follow immediately, and the solution $u(x)$ can be obtained using (2).

It is evident that algorithm (10) reduces the number of terms involved in each component, and hence the size of calculations is minimized compared to the standard Adomian decomposition method. Moreover, this reduction of terms in each component facilitates the construction of Adomian polynomials for nonlinear operators [5].

The New Modified Decomposition Method. As indicated earlier, MADM1 may provide the exact solution by using two iterations only and sometimes without any need for Adomian polynomials, but its effectiveness depends on the proper choice of $f_{1}(x)$ and $f_{2}(x)$, which may need quite a little computational work. Regarding SADM and MADM2, sometimes one will inevitably encounter the difficulties as will be seen 
from the examples below. In this new modification, we obtain the first two terms by

$$
\begin{gathered}
u_{0}(x)=f(x) \\
u_{1}(x)=\lambda \int_{a}^{x} K(x, t)\left(L\left(u_{0}\right)+A_{0}\right) \mathrm{d} t
\end{gathered}
$$

which are the same as the results by SADM. However, sometimes $u_{1}(x)$ is too complicated to continue or is analytically impossible. Here, we suggest that $u_{1}(x)$ may be expressed in Taylor series form as

$$
u_{1}(x)=\sum_{i=0}^{+\infty} u_{1 i}(x)
$$

next, we set

$$
u_{2}(x)=\lambda \int_{a}^{x} K(x, t)\left(L\left(u_{1}\right)+A_{1}\right) \mathrm{d} t
$$

where the Adomian polynomial $A_{1}$ can be evaluated by (4) with $u_{0}(x)$ and $u_{1}(x)$, which will be obtained by (11a) and (12), respectively. Also, we suggest that the same series expansion can be acted upon $u_{2}(x)$, that is,

$$
u_{2}(x)=\sum_{i=0}^{+\infty} u_{2 i}(x)
$$

Similarly, we set

$$
u_{k}(x)=\lambda \int_{a}^{x} K(x, t)\left(L\left(u_{k}\right)+A_{k}\right) \mathrm{d} t, \quad k \geq 3,
$$

and their Taylor expansions given by

$$
u_{k}(x)=\sum_{i=0}^{+\infty} u_{k i}(x), \quad k \geq 3 .
$$

By combining (11a), (12), (14), and (16), the solution $u(x)$ can be obtained using the series (2).

In practice, for some problems, we have to determine a few terms in the series such as $u(x)=\sum_{i=0}^{M} u_{i}(x)$ by truncating the series at certain term, regardless of the standard decomposition method and the modified decomposition method. Because of the uniformly convergence property of the infinite series, a few terms will attain the maximum accuracy.

To give a clear overview of this study, we have chosen several linear and nonlinear Volterra integral equations to testify the validity and applicability of the new modification. For comparison, examples will also be discussed by using SADM, MADM1, and MADM2.

\section{Application for Linear Integral Equations}

Example 1. Consider the linear Volterra integral equation [12]

$$
u(x)=\sin x+e-e^{\cos x}-\int_{0}^{x} e^{\cos t} u(t) \mathrm{d} t
$$

Following suggestions (6a) and (6b) of SADM, the second component $u_{1}(x)$ of the solution $u(x)$ cannot be obtained because of the impossible integration, which also appears by using $M A D M 2$. If we turn to $M A D M 1$, the six different choices of $f_{1}(x)$ and $f_{2}(x)$ need quite a little computational work only if we set $f_{1}(x)=\sin x$ and $f_{2}(x)=e-e^{\cos x}$ directly. In the following part, we solve (17) by the new modification with the help of computer algebraic system Maple 12.

According to suggestions (11a) and (11b), we obtain

$$
\begin{gathered}
u_{0}(x)=\sin x+e-e^{\cos x}, \\
u_{1}(x)=-\int_{0}^{x} e^{\cos t} u_{0}(t) \mathrm{d} t .
\end{gathered}
$$

We then set the Taylor expression for $u_{1}(x)$ by

$$
\begin{aligned}
u_{1}(x)= & -\frac{1}{2} e x^{2}-\frac{1}{6} e^{2} x^{3}+\frac{1}{6} e x^{4} \\
& +\frac{1}{12} e^{2} x^{5}-\frac{31}{720} e x^{6}-\cdots
\end{aligned}
$$

And also, we set the Taylor expansion for $u_{i}(x), i=2,3,4, \ldots$ because the evaluation of integrals, obtained by applying (13) and (15) in this example, is analytically impossible. In this way, we have

$$
\begin{gathered}
u_{2}(x)=-\int_{0}^{x} e^{\cos t} u_{1}(t) \mathrm{d} t \\
=\frac{1}{6} e^{2} x^{3}+\frac{1}{24} e^{3} x^{4}-\frac{1}{12} e^{2} x^{5}-\frac{1}{36} e^{3} x^{6}+\cdots, \\
\begin{aligned}
& u_{3}(x)=-\int_{0}^{x} e^{\cos t} u_{2}(t) \mathrm{d} t \\
&=-\frac{1}{24} e^{3} x^{4}-\frac{1}{120} e^{4} x^{5}+\frac{1}{36} e^{3} x^{6}+\cdots, \\
& u_{4}(x)=-\int_{0}^{x} e^{\cos t} u_{3}(t) \mathrm{d} t \\
&=\frac{1}{120} e^{4} x^{5}+\frac{1}{720} e^{5} x^{6}-\cdots \\
& u_{5}(x)=-\int_{0}^{x} e^{\cos t} u_{4}(t) \mathrm{d} t \\
&=-\frac{1}{720} e^{5} x^{6}-\cdots
\end{aligned}
\end{gathered}
$$

Thus, the solution in a series form is given by

$$
u(x)=\sin x+e-e^{\cos x}+e\left(-\frac{1}{2} x^{2}+\frac{1}{6} x^{4}-\frac{31}{720} x^{6}+\cdots\right) .
$$

Combining (21) with the Taylor expansion of

$$
e-e^{\cos x}=e\left(\frac{1}{2} x^{2}-\frac{1}{6} x^{4}+\frac{31}{720} x^{6}-\cdots\right),
$$


the solution in a closed form is given by

$$
u(x)=\sin x
$$

Example 2. Consider the linear Volterra integral equation [5, 13]

$$
u(x)=1+\sinh x-\cosh x+\int_{0}^{x} u(t) \mathrm{d} t
$$

In this example, both $S A D M$ and MADM2 can fulfil the exact solution effectively. Whereas, MADM1 needs quite a little computational work to obtain the exact solution only if we set $f_{1}(x)=\sinh x$ and $f_{2}(x)=1-\cosh x$ directly. In the following part, we solve (24) by the new modification.

Proceeding as before, we set

$$
\begin{gathered}
u_{0}(x)=1+\sinh x-\cosh x \\
u_{1}(x)=\int_{0}^{x} u_{0}(t) \mathrm{d} t=\cosh x-\sinh x+x-1 .
\end{gathered}
$$

We then set the Taylor expression for $u_{1}(x)$ by

$$
u_{1}(x)=\frac{1}{2 !} x^{2}-\frac{1}{3 !} x^{3}+\frac{1}{4 !} x^{4}-\frac{1}{5 !} x^{5}+\frac{1}{6 !} x^{6}-\cdots,
$$

and also

$$
\begin{aligned}
u_{2}(x) & =\int_{0}^{x} u_{1}(t) \mathrm{d} t \\
& =\frac{1}{3 !} x^{3}-\frac{1}{4 !} x^{4}+\frac{1}{5 !} x^{5}-\frac{1}{6 !} x^{6}+\frac{1}{7 !} x^{7}-\cdots, \\
u_{3}(x) & =\int_{0}^{x} u_{2}(t) \mathrm{d} t \\
& =\frac{1}{4 !} x^{4}-\frac{1}{5 !} x^{5}+\frac{1}{6 !} x^{6}-\frac{1}{7 !} x^{7}+\frac{1}{8 !} x^{8}-\cdots, \\
u_{4}(x) & =\int_{0}^{x} u_{3}(t) \mathrm{d} t \\
& =\frac{1}{5 !} x^{5}-\frac{1}{6 !} x^{6}+\frac{1}{7 !} x^{7}-\frac{1}{8 !} x^{8}+\frac{1}{9 !} x^{9}-\cdots, \\
u_{5} & (x)=\int_{0}^{x} u_{4}(t) \mathrm{d} t \\
& =\frac{1}{6 !} x^{6}-\frac{1}{7 !} x^{7}+\frac{1}{8 !} x^{8}-\frac{1}{9 !} x^{9}+\cdots,
\end{aligned}
$$

The solution in a series form is given by

$$
u(x)=\sinh x-\cosh x+\left(1+\frac{1}{2 !} x^{2}+\frac{1}{4 !} x^{4}+\frac{1}{6 !} x^{6}+\cdots\right),
$$

and in a closed form by

$$
u(x)=\sinh x .
$$

Example 3. Consider the system of linear Volterra integral equations [12]

$$
\begin{aligned}
u(x)= & \cos x-x \sin x \\
& +\int_{0}^{x}(\sin (x-t) u(t)+\cos (x-t) v(t)) \mathrm{d} t, \\
v(x)= & \sin x-x \cos x \\
& +\int_{0}^{x}(\cos (x-t) u(t)-\sin (x-t) v(t)) \mathrm{d} t .
\end{aligned}
$$

In this example, it is both difficult to obtain the exact solution by using SADM and MADM2. And also, like the problem mentioned in preceding examples, MADM1 needs much computational work to obtain the exact solution only if we set $f_{1}(x)=\cos x$ and $f_{2}(x)=-x \sin x$ for the first equation and $f_{1}(x)=\sin x$ and $f_{2}(x)=-x \cos x$ for the second equation, directly. In the following parts, we solve (30) by the new modification.

Proceeding as before, the system (30) is transformed into a set of recursive relations given by

$$
\begin{gathered}
u_{0}(x)=\cos x-x \sin x, \\
v_{0}(x)=\sin x-x \cos x, \\
u_{1}(x)=\int_{0}^{x}\left(\sin (x-t) u_{0}(t)+\cos (x-t) v_{0}(t)\right) \mathrm{d} t \\
v_{1}(x)=\int_{0}^{x}\left(\cos (x-t) u_{0}(t)-\sin (x-t) v_{0}(t)\right) \mathrm{d} t .
\end{gathered}
$$

We then set the Taylor expansion for $u_{1}(x)$ and $v_{1}(x)$ by

$$
\begin{gathered}
u_{1}(x)=\frac{1}{2} x^{2}-\frac{1}{12} x^{4}+\frac{1}{240} x^{6}-\cdots, \\
v_{1}(x)=x-\frac{2}{3} x^{3}+\frac{7}{120} x^{5}-\cdots .
\end{gathered}
$$

Similarly,

$$
\begin{aligned}
u_{2}(x) & =\int_{0}^{x}\left(\sin (x-t) u_{1}(t)+\cos (x-t) v_{1}(t)\right) \mathrm{d} t \\
& =\frac{1}{2} x^{2}-\frac{1}{6} x^{4}+\frac{1}{80} x^{6}-\cdots, \\
v_{2}(x) & =\int_{0}^{x}\left(\cos (x-t) u_{1}(t)-\sin (x-t) v_{1}(t)\right) \mathrm{d} t \\
& =\frac{1}{60} x^{5}-\frac{1}{840} x^{7}+\cdots,
\end{aligned}
$$




$$
\begin{aligned}
u_{3}(x) & =\int_{0}^{x}\left(\sin (x-t) u_{2}(t)+\cos (x-t) v_{2}(t)\right) \mathrm{d} t \\
& =\frac{1}{24} x^{4}-\frac{1}{240} x^{6}+\cdots, \\
v_{3}(x) & =\int_{0}^{x}\left(\cos (x-t) u_{2}(t)-\sin (x-t) v_{2}(t)\right) \mathrm{d} t \\
& =\frac{1}{6} x^{3}-\frac{1}{24} x^{5}+\frac{1}{420} x^{7}-\cdots, \\
u_{4}(x) & =\int_{0}^{x}\left(\sin (x-t) u_{3}(t)+\cos (x-t) v_{3}(t)\right) \mathrm{d} t \\
& =\frac{1}{24} x^{4}-\frac{1}{144} x^{6}+\cdots, \\
v_{4}(x) & =\int_{0}^{x}\left(\cos (x-t) u_{3}(t)-\sin (x-t) v_{3}(t)\right) \mathrm{d} t \\
u_{6}(x) & =\int_{0}^{x}\left(\sin (x-t) u_{5}(t)+\cos (x-t) v_{5}(t)\right) \mathrm{d} t \\
& =\frac{1}{720} x^{6}-\frac{1}{6720} x^{8}+\cdots, \\
& =\frac{1}{2520} x^{7}-\frac{1}{45360} x^{9}+\cdots, \\
& =\frac{1}{720} x^{6}-\frac{1}{10080} x^{8}+\cdots, \\
u_{5}(x) & =\int_{0}^{x}\left(\sin (x-t) u_{4}(t)+\cos (x-t) v_{4}(t)\right) \mathrm{d} t
\end{aligned}
$$

The solution in a series form is given by

$$
\begin{aligned}
& u(x)=\cos x-x \sin x+x\left(x-\frac{1}{6} x^{3}+\frac{1}{120} x^{5}-\cdots\right) \\
& v(x)=\sin x-x \cos x+x\left(1-\frac{1}{2} x^{2}+\frac{1}{24} x^{4}-\cdots\right)
\end{aligned}
$$

and in a closed form by

$$
(u(x), v(x))=(\cos x, \sin x) .
$$

\section{Application for Nonlinear Integral Equations}

Example 4. Consider the nonlinear Volterra integral equation $[12,14]$

$$
u(x)=e^{x}-\frac{1}{3} x e^{3 x}+\frac{1}{3} x+\int_{0}^{x} x u^{3}(t) \mathrm{d} t
$$

Following suggestions (6a) and (6b) of SADM, the second component $u_{1}(x)$ described via the sum of twenty-seven terms is too complicated for no confidence to continue. Meanwhile, there does not exist noise terms [15], which leads to the result quickly. By $M A D M 1$, the six different choices of $f_{1}(x)$ and $f_{2}(x)$ need quite a little computational work as the examples given before, unless we set $f_{1}(x)=e^{x}$ and $f_{2}(x)=-(1 / 3) x e^{3 x}+(1 / 3) x$ directly. The solution can be obtained by MADM2 exactly. In the following part, we solve (36) by the new modification.

According to suggestions (11a) and (11b), we obtain

$$
\begin{gathered}
u_{0}(x)=e^{x}-\frac{1}{3} x e^{3 x}+\frac{1}{3} x \\
u_{1}(x)=\int_{0}^{x} x u_{0}^{3}(t) \mathrm{d} t
\end{gathered}
$$

We then set the Taylor expression for $u_{1}(x)$ by

$$
u_{1}(x)=x^{2}+\frac{3}{2} x^{3}+\frac{1}{2} x^{4}-\frac{3}{2} x^{5}-\frac{21}{8} x^{6}-\frac{227}{120} x^{7}+\cdots
$$

And also, we set the Taylor expansion for $u_{i}(x), i=2,3,4, \ldots$ because of the complicated integration. In this way, we have

$$
\begin{aligned}
u_{2}(x) & =3 \int_{0}^{x} x u_{0}^{2}(t) u_{1}(t) \mathrm{d} t \\
& =x^{4}+\frac{21}{8} x^{5}+\frac{21}{10} x^{6}-\frac{25}{12} x^{7}-\cdots, \\
u_{3}(x) & =3 \int_{0}^{x} x\left(u_{0}^{2}(t) u_{2}(t)+u_{0}(t) u_{1}^{2}(t)\right) \mathrm{d} t \\
& =\frac{6}{5} x^{6}+\frac{69}{16} x^{7}+\cdots,
\end{aligned}
$$

where the Adomian polynomials for nonlinear term $u^{3}$ were calculated by using (4). The solution in a series form is given by

$$
\begin{aligned}
u(x)= & \left(e^{x}-\frac{1}{3} x e^{3 x}+\frac{1}{3} x\right) \\
& +\left(x^{2}+\frac{3}{2} x^{3}+\frac{3}{2} x^{4}+\frac{9}{8} x^{5}+\frac{27}{40} x^{6}+\frac{27}{80} x^{7}+\cdots\right) .
\end{aligned}
$$

Combining (40) with the Taylor expansion of

$$
\begin{aligned}
-\frac{1}{3} x e^{3 x}+\frac{1}{3} x= & -x^{2}-\frac{3}{2} x^{3}-\frac{3}{2} x^{4} \\
& -\frac{9}{8} x^{5}-\frac{27}{40} x^{6}-\frac{27}{80} x^{7}-\cdots,
\end{aligned}
$$

the solution in a closed form is given by

$$
u(x)=e^{x} .
$$


Example 5. Consider the nonlinear Volterra integrodifferential equation of the first kind [12]

$$
\begin{gathered}
\int_{0}^{x}(x-t) u^{2}(t) \mathrm{d} t+\int_{0}^{x} e^{x-t} u^{\prime}(t) \mathrm{d} t=x e^{x}+\frac{1}{4} e^{2 x}-\frac{1}{4}-\frac{1}{2} x, \\
u(0)=1 .
\end{gathered}
$$

By integrating the second integral by parts and using the initial conditions, (43) can be converted to Volterra integral equation of the second kind given by

$$
\begin{aligned}
u(x)= & e^{x}+x e^{x}+\frac{1}{4} e^{2 x}-\frac{1}{4}-\frac{1}{2} x \\
& -\int_{0}^{x}\left((x-t) u^{2}(t)+e^{x-t} u(t)\right) \mathrm{d} t .
\end{aligned}
$$

Following suggestions (6a) and (6b) of SADM, $u_{1}(x)$ consists of fourteen terms and $u_{2}(x)$ twenty-nine terms. It is so complicated that we can give up. The similar obstacle derived from the selections of $f_{1}(x)$ and $f_{2}(x)$ will be encountered if we use MADM1. By using MADM2, it is of certain difficulty to obtain the exact solution through the first finite terms of the series solution. In the following part, we solve (43) by the new modification.

Following as before, we set

$$
\begin{gathered}
u_{0}(x)=e^{x}+x e^{x}+\frac{1}{4} e^{2 x}-\frac{1}{4}-\frac{1}{2} x, \\
u_{1}(x)=-\int_{0}^{x}\left((x-t) u_{0}^{2}(t)+e^{x-t} u_{0}(t)\right) \mathrm{d} t \\
=-x-2 x^{2}-\frac{11}{6} x^{3}-\frac{29}{24} x^{4}-\frac{41}{60} x^{5}-\cdots, \\
u_{2}(x)=-\int_{0}^{x}\left(2(x-t) u_{0}(t) u_{1}(t)+e^{x-t} u_{1}(t)\right) \mathrm{d} t \\
=\frac{1}{2} x^{2}+\frac{7}{6} x^{3}+\frac{4}{3} x^{4}+\cdots, \\
u_{3}(x)=-\int_{0}^{x}\left((x-t)\left(2 u_{0}(t) u_{2}(t)+u_{1}^{2}(t)\right)\right. \\
\left.u_{4}(x)=-\frac{1}{24} x^{4}+\cdots, \quad+e^{x-t} u_{2}(t)\right) \mathrm{d} t \\
=-\frac{1}{6} x^{3}-\frac{1}{2} x^{4}-\cdots, \\
\quad(x-t)\left(e_{0}^{x-t} u_{3}(t)\right) \mathrm{d} t \\
\left.=u_{3}(t)+u_{1}(t) u_{2}(t)\right)
\end{gathered}
$$

where Adomian polynomials for nonlinear term $u^{2}$ were calculated by using (4). The solution in a series form is given by

$$
\begin{aligned}
u(x)= & \left(e^{x}+x e^{x}+\frac{1}{4} e^{2 x}\right) \\
& +\left(-\frac{1}{4}-\frac{3}{2} x-\frac{3}{2} x^{2}-\frac{5}{6} x^{3}-\frac{1}{3} x^{4}-\cdots\right)
\end{aligned}
$$

Combining (46) with the Taylor expansion of

$$
x e^{x}+\frac{1}{4} e^{2 x}=\frac{1}{4}+\frac{3}{2} x+\frac{3}{2} x^{2}+\frac{5}{6} x^{3}+\frac{1}{3} x^{4}+\cdots,
$$

the solution in a closed form is given by

$$
u(x)=e^{x} .
$$

\section{Discussion and Conclusion}

In this work, we have carefully developed a new modification of Adomian decomposition method based on the Taylor expansion of the components apart from the zeroth term for Volterra integral equations. The new technology overcomes such obstacles as the complicated integrals and the impossible integrals analytically, which arise from either $S A D M$ or $M A D M 2$. If the exact solution exists in the zeroth component, $M A D M 1$ needs extensive workload for the suitable choice of $f_{1}(x)$ and $f_{2}(x)$, while the new modification can proceed in a fixed manner. Therefore, it is appropriate for one to implement through the mathematical software Maple without manual tedious calculations.

The idea has been shown to be computationally efficient in applying the proposed technique to several linear and nonlinear Volterra integral equations and integrodifferential equations. Also, through the examples, it is seen that the new modification suits for those integral equations where the exact solution appears as part of the zeroth term. It is also interesting to point out that one can obtain the approximate solution of integral equations as mentioned in $[16,17]$ simply by slightly varying the new modification of this work.

\section{Acknowledgment}

The work was supported by the Scientific Research Fund of Zhejiang Provincial Education Department of China (no. Y201120655), K.C. Wong Magna Fund in Ningbo University.

\section{References}

[1] G. Adomian, "A review of the decomposition method and some recent results for nonlinear equations," Mathematical and Computer Modelling, vol. 13, no. 7, pp. 17-43, 1990.

[2] G. Adomian, Solving Frontier Problems of Physics: The Decomposition Method, vol. 60 of Fundamental Theories of Physics, Kluwer Academic, Dordrecht, The Netherlands, 1994.

[3] Y. Cherruault, G. Saccomandi, and B. Some, "New results for convergence of Adomian's method applied to integral equations," Mathematical and Computer Modelling, vol. 16, no. 2, pp. 85-93, 1992. 
[4] A.-M. Wazwaz, "A reliable modification of Adomian decomposition method," Applied Mathematics and Computation, vol. 102, no. 1, pp. 77-86, 1999.

[5] A.-M. Wazwaz and S. M. El-Sayed, "A new modification of the Adomian decomposition method for linear and nonlinear operators," Applied Mathematics and Computation, vol. 122, no. 3, pp. 393-405, 2001.

[6] M. M. Hosseini, "Adomian decomposition method with Chebyshev polynomials," Applied Mathematics and Computation, vol. 175, no. 2, pp. 1685-1693, 2006.

[7] Y. Liu, "Adomian decomposition method with orthogonal polynomials: Legendre polynomials," Mathematical and Computer Modelling, vol. 49, no. 5-6, pp. 1268-1273, 2009.

[8] W.-C. Tien and C.-K. Chen, "Adomian decomposition method by Legendre polynomials," Chaos, Solitons \& Fractals, vol. 39, no. 5, pp. 2093-2101, 2009.

[9] Y. Çenesiz and A. Kurnaz, "Adomian decomposition method by Gegenbauer and Jacobi polynomials," International Journal of Computer Mathematics, vol. 88, no. 17, pp. 3666-3676, 2011.

[10] A.-M. Wazwaz, "A new algorithm for calculating Adomian polynomials for nonlinear operators," Applied Mathematics and Computation, vol. 111, no. 1, pp. 33-51, 2000.

[11] E. Babolian and Sh. Javadi, "New method for calculating Adomian polynomials," Applied Mathematics and Computation, vol. 153, no. 1, pp. 253-259, 2004.

[12] A.-M. Wazwaz, Linear and Nonlinear Integral Equations: Methods and Applications, Higher Education Press, Beijing, China, 2011.

[13] A.-M. Wazwaz, A First Course in Integral Equations, World Scientific Publishing, River Edge, NJ, USA, 1997.

[14] M. Rahman, Integral Equations and Their Applications, WIT Press, Southampton, UK, 2007.

[15] A.-M. Wazwaz and A. M. Wazwaz, "Necessary conditions for the appearance of noise terms in decomposition solution series," Applied Mathematics and Computation, vol. 81, no. 2-3, pp. 265274, 1997.

[16] E. Babolian and A. Davari, "Numerical implementation of Adomian decomposition method," Applied Mathematics and Computation, vol. 153, no. 1, pp. 301-305, 2004.

[17] E. Babolian and A. Davari, "Numerical implementation of Adomian decomposition method for linear Volterra integral equations of the second kind," Applied Mathematics and Computation, vol. 165, no. 1, pp. 223-227, 2005. 


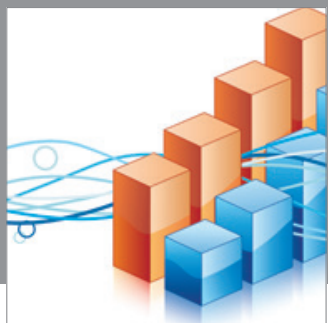

Advances in

Operations Research

mansans

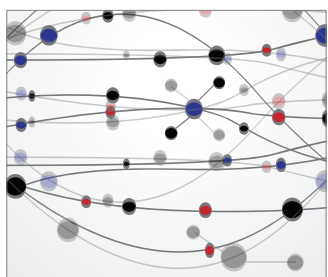

The Scientific World Journal
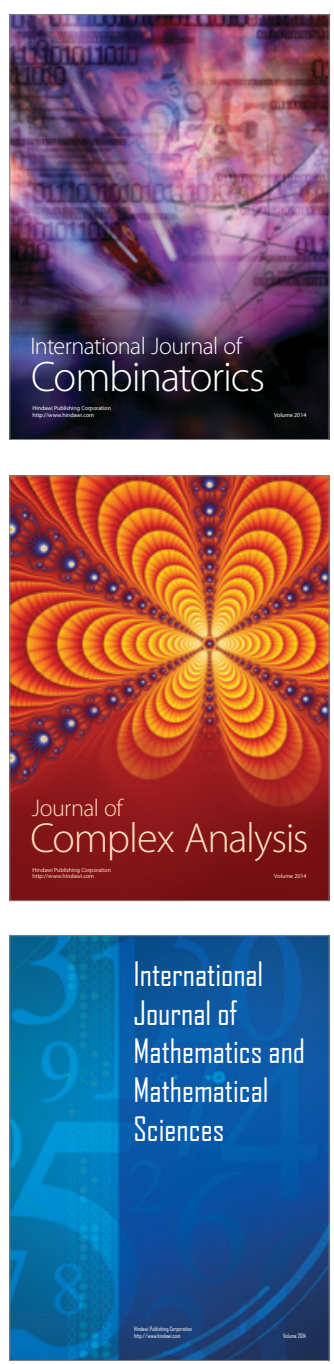
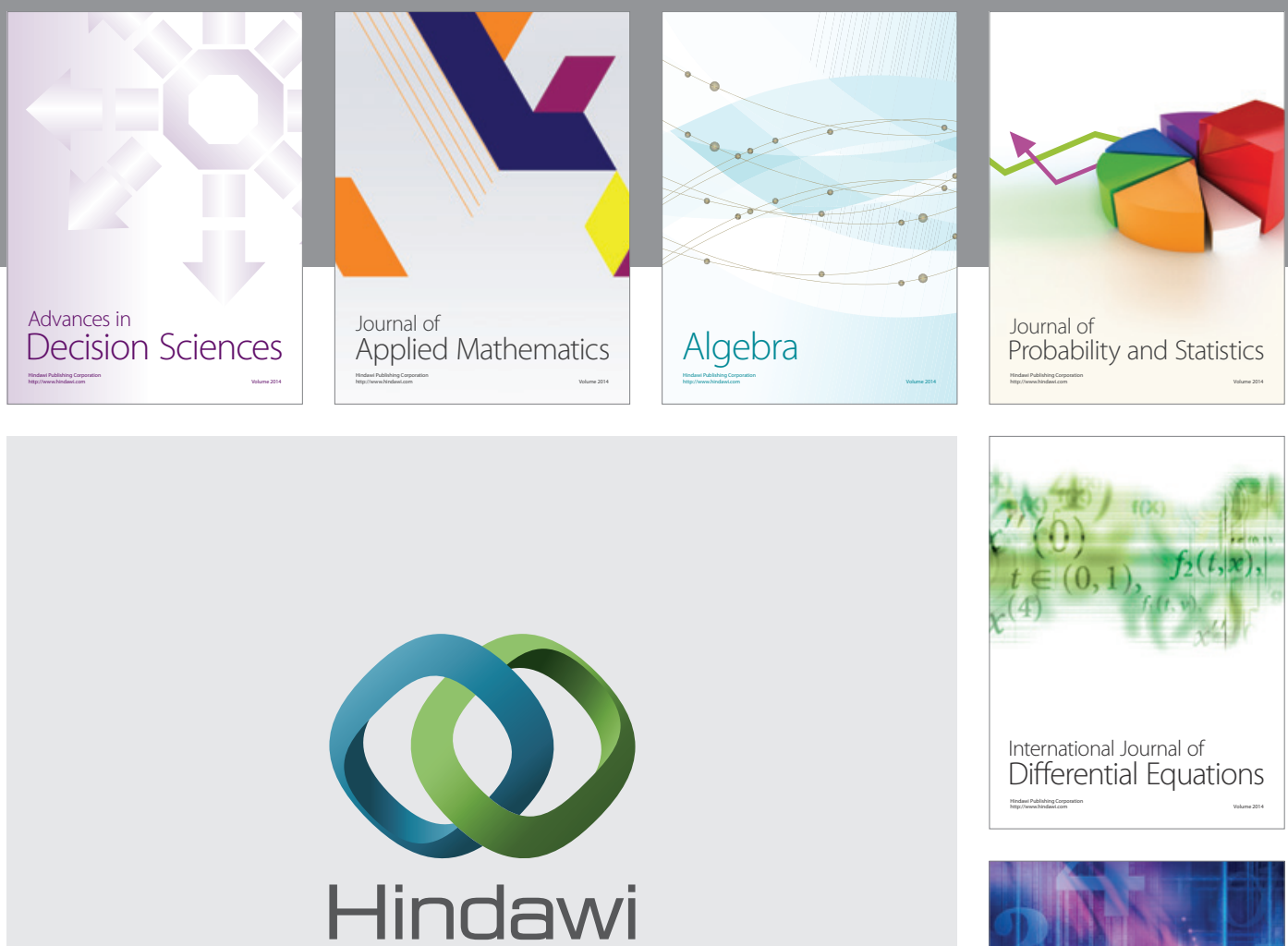

Submit your manuscripts at http://www.hindawi.com
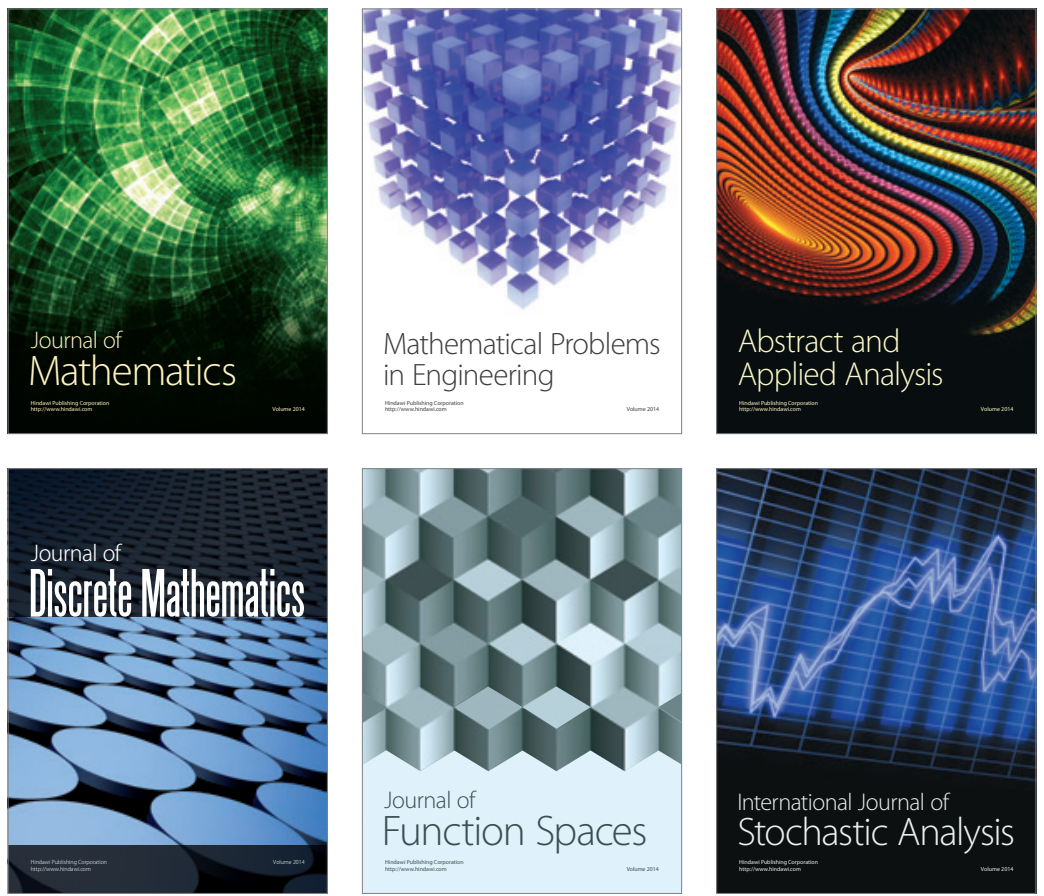

Journal of

Function Spaces

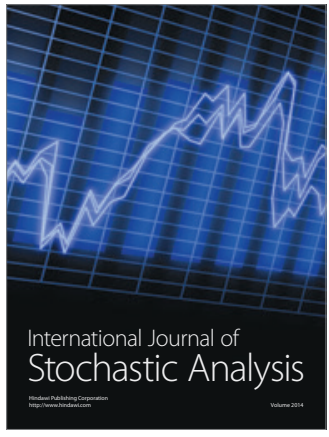

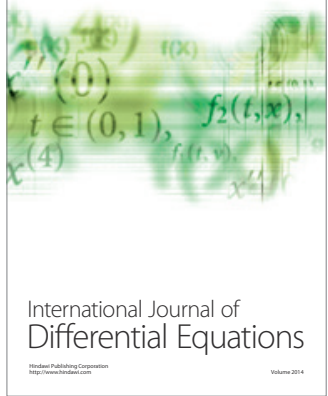
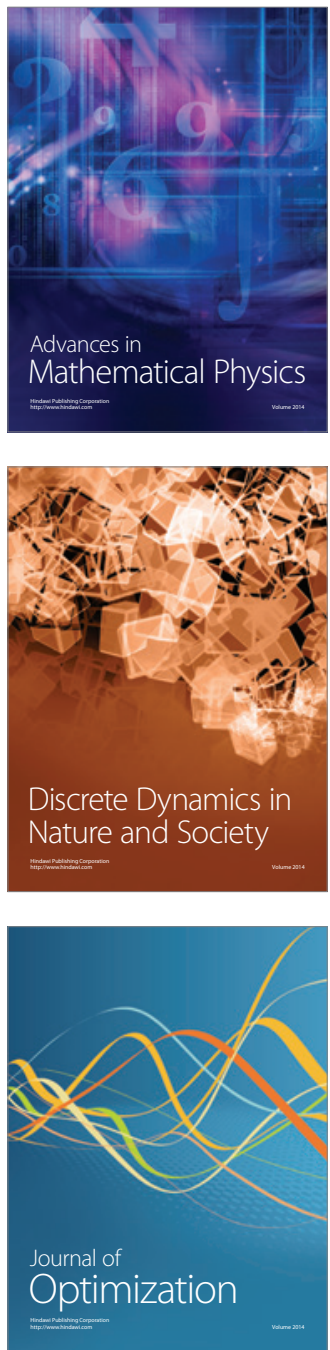